\title{
Avaliação dos resultados funcionais do tratamento cirúrgico artroscópico da lesão completa do manguito rotador com seguimento mínimo de 10 anos*
}

\section{Evaluation of the Functional Outcomes of Arthroscopic Surgical Treatment of Complete Rotator Cuff Lesion with Minimum Follow-up of 10 Years}

\author{
André Couto Godinho ${ }^{10} \quad$ Flávio Márcio Lago Santos ${ }^{2(10}$ Francisco Pereira Donato Neto ${ }^{10}$ \\ Paulo Victor Nunes Paz Silva ${ }^{10}$ Ricardo Dantas Fonseca Júnior ${ }^{10}$
}

${ }^{1}$ Serviço de Ombro do Hospital Ortopédico BH, Belo Horizonte, MG, Brasil

2 Serviço de Ombro do Hospital Lifecenter, Belo Horizonte, MG, Brasil

Endereço para correspondência André Couto Godinho, MSc, Rua dos Aimorés, 2085, Apartamento 1502, Belo Horizonte, MG, 30140-072, Brasil (e-mail: andre_cgod@hotmail.com).

Rev Bras Ortop 2020;55(5):579-584.

\section{Resumo \\ Palavras-chave \\ - ombro \\ - artroscopia \\ - ruptura \\ - manguito rotador \\ - estudos de avaliação \\ Objetivo Analisar o resultado funcional dos pacientes submetidos ao tratamento cirúrgico videoartroscópico da ruptura completa do manguito rotador do ombro, com seguimento mínimo de 10 anos. \\ Métodos Foram avaliados 63 pacientes ( 63 ombros) submetidos ao reparo cirúrgico videoartroscópico da ruptura completa do manguito rotador com seguimento mínimo de 10 anos. $O$ resultado funcional no pós-operatório desses pacientes foi avaliado pelos escores de Constant e UCLA. \\ Resultados Os valores médios foram de 26 pontos no escore UCLA e de 93 no escore de Constant. Para o escore de Constant, $91 \%$ foram considerados satisfatórios; e para o UCLA, 62\% dos pacientes apresentaram escores satisfatórios. \\ Conclusão O reparo artroscópico da ruptura completa do manguito rotador mostrou- se efetivo mesmo a longo prazo (seguimento mínimo de dez anos). A idade dos pacientes antes da cirurgia, o tamanho da lesão, o grau de infiltração gordurosa e a avaliação do trofismo muscular foram importantes preditores de prognóstico.}

Abstract
Objectives To analyze the functional outcome of patients submitted to videoarthroscopic surgical treatment for compleat rotator cuff tears of the shoulder, with a minimum follow-up of 10 years.

Methods A total of 63 patients (63 shoulders) underwent videoarthroscopic surgical repair of compleat rotator cuff tears with a minimum follow-up of 10 years. The

Trabalho realizado pelo Grupo de Cirurgia e Reabilitação do Ombro de Belo Horizonte.

recebido

10 de Outubro de 2018 aceito

26 de Fevereiro de 2019
DOI https://doi.org/

10.1055/s-0040-1715510. ISSN 0102-3616.
Copyright $\odot 2020$ by Sociedade Brasileira License terms de Ortopedia e Traumatologia. Published by Thieme Revinter Publicações Ltda, Rio de Janeiro, Brazil 
Keywords

- shoulder

- arthroscopy

- rupture

- rotator cuff

- evaluation studies postoperative functional outcomes of these patients were evaluated using the Constant and University of California at Los Angeles (UCLA) scores.

Results The functional evaluation revealed mean UCLA and Constant scores of 26 and 93 points, respectively. Ninety-one percent of the subjects had satisfactory Constant scores, whereas $62 \%$ presented satisfactory UCLA scores.

Conclusion The arthroscopic repair of the rotator cuff complete tear was effective even in the long term (minimum follow-up period of 10 years). The age of the patients before surgery, size of the lesion, the degree of fatty infiltration, and evaluation of muscle trophism are important predictors of prognosis.

\section{Introdução}

As lesões degenerativas e traumáticas que afetam o manguito rotador (MR) estão entre as doenças mais comuns do ombro, principalmente na população feminina acima dos 50 anos de idade. ${ }^{1}$

A patogênese da lesão do manguito rotador é ainda obscura, mas acredita-se que ela seja multifatorial, resultando de avascularidade, idade, ou carga excêntrica, sendo estes fatores associados à falência de suas fibras e consequente prejuízo funcional. ${ }^{2}$ Em alguns casos, as rupturas podem ser assintomáticas; porém, a doença tem caráter progressivo e degenerativo, o que pode causar dor e comprometer a função do ombro. ${ }^{3}$

0 diagnóstico é essencialmente clínico, através de anamnese detalhada e do exame físico minucioso. ${ }^{4}$ Alguns exames de imagem, principalmente a ressonância magnética (RM), auxiliam no diagnóstico. ${ }^{5}$

O tratamento consiste desde a forma não cirúrgica, incluindo terapia farmacológica e fortalecimento muscular, até o reparo cirúrgico, seja ele por via aberta ou artroscópica, ambos apresentando bons resultados. ${ }^{4}$

O tratamento cirúrgico das lesões do manguito rotador (LMR) tem sido cada vez mais indicado, e a técnica artroscópica é a mais difundida nos últimos anos por ser pouco agressiva ao músculo deltoide, menos dolorosa e, consequentemente, permitindo uma reabilitação mais precoce quando comparada ao reparo tendinoso aberto. ${ }^{6}$ Esta técnica tem como vantagens: avaliação completa da articulação glenoumeral e lesões associadas (lesões condrais, artrose glenoumeral, artrose acromioclavicular, tenossinovite da cabeça longa do bíceps, lesões capsuloligamentares, lesões SLAP (superior labral anterior and posterior"), etc...7,8

O objetivo do presente trabalho foi analisar o resultado funcional dos pacientes submetidos ao tratamento cirúrgico videoartroscópico da ruptura completa do manguito rotador do ombro, por meio dos escores citados acima e com seguimento mínimo de 10 anos após a realização do tratamento cirúrgico.

\section{Casuística e métodos}

O presente trabalho trata-se de um estudo de avaliação retrospectiva no qual foram incluídos 100 pacientes, selecionados de forma aleatória, que tiveram o manguito rotador reparado por via artroscópica, com um seguimento mínimo de 10 anos. Os mesmos foram operados pelos cirurgiões de ombro de um mesmo grupo, executando técnica cirúrgica padrão.

Todos os pacientes foram submetidos ao tratamento cirúrgico da lesão do manguito rotador na posição decúbito lateral, por via artroscópica com a técnica de sutura tendínea em fileira simples utilizando âncoras metálicas ${ }^{9}$, associada ou não a tenotomia e/ou tenodese do tendão da cabeça longa do bíceps (TCLB), acromioplastia e procedimento de Mumford total (ressecção total da extremidade lateral da clavícula). ${ }^{10}$

O Mumford total artroscópico foi realizado em pacientes com o diagnóstico de artrose da articulação acromioclavicular sintomática, confirmado por achados radiográficos (esclerose, cistos subcondrais, irregularidade articular), RM demonstrando presença de edema na extremidade lateral da clavícula, dor à palpação da articulação, teste de adução cruzada positivo, ${ }^{11}$ e teste de $0^{\prime} B r i e n$ positivo. ${ }^{12}$

Os pacientes compareceram espontaneamente para se submeterem à avaliação clínica e radiográfica realizada por três ortopedistas especializando-se em cirurgia de ombro. Foram excluídos aqueles com diagnóstico prévio de osteoartrite glenoumeral, artrite reumatoide, sequelas de fraturas do úmero proximal, instabilidade glenoumeral associada e documentação incompleta. Após a aplicação dos critérios de exclusão, restaram 63 pacientes (63 ombros), que constituem a série de casos em questão.

Fatores pré-operatórios, tais como o grau de infiltração gordurosa utilizando a classificação de Goutallier et al., ${ }^{13}$ foram agrupados em: grupo 0 (músculo sem infiltração gordurosa), grupo 1 (inclui os graus 1 e 2-infiltração de baixo grau) e, grupo 2 (inclui os graus 3 e 4 -infiltração de alto grau). Considerou-se também idade, sexo, lado acometido, tabagismo, dominância, mecanismo da lesão, e trofismo muscular caracterizado pelo sinal da tangente de Zannetti et al. ${ }^{14}$ (linha traçada unindo a extremidade da apófise coracoide à espinha da escápula no corte sagital oblíquo da RM, a qual determina o trofismo do músculo supraespinal que, se situado abaixo da mesma, indica hipotrofia e, acima dela, eutrofia) e a extensão anteroposterior das lesões de acordo com a classificação de DeOrio e Cofield. ${ }^{15}$

O tempo de seguimento pós-operatório variou entre 10 e 18 anos (mínimo de 10 anos e máximo de 18 anos, com uma média de 14,2 anos). Trinta e seis pacientes eram do sexo feminino (57,1\%) e 27 do sexo masculino (42,9\%). A média de 
idade foi de 57,2 anos (mínima de 46 anos e máxima de 72 anos). 0 lado dominante foi acometido em 46 pacientes (73\%). A lesão de etiologia degenerativa correspondeu a $82,5 \%$ da amostra e a de origem traumática correspondeu a cerca de $17,5 \%$.

Utilizamos os escores de Constant ${ }^{16}$ e UCLA $^{17}$ para avaliar o resultado funcional no pós-operatório desses pacientes, fazendo o cruzamento destes com os dados pré-operatórios.

A integridade do reparo no pós-operatório tardio foi avaliada por meio de exames de RM realizados em todos os pacientes que constituem a série de casos em questão. Foi classificado como re-ruptura do manguito rotador a ausência de continuidade do tendão em seu trajeto da junção miotendínea até a inserção no tubérculo maior.

Para a análise descritiva dos dados, utilizou-se a média e o desvio-padrão (variáveis quantitativas), frequência absoluta e porcentagens (variáveis qualitativas). Para testar diferenças entre grupos em relação às variáveis quantitativas, utilizou-se o teste $t$ de Student para amostras independentes. Para testar a associação entre as classificações funcionais (satisfatório e insatisfatório) e possíveis variáveis explicativas, utilizou-se o teste do Qui-quadrado ou o teste exato de Fisher, quando apropriado. Para as variáveis quantitativas, o tamanho do efeito foi avaliado pelo $d$ de Cohen, sendo adotada a seguinte classificação para interpretação: pequeno: $0,2-0,4$; moderado: $0,5-0,7$; elevado: $\geq 0,8$; para as variáveis qualitativas, utilizou-se o $V$ de Cramer, sendo adotada a seguinte classificação para interpretação: pequeno: $0,1-0,2$; moderado: 0,3-0,4; elevado: $\geq 0,5$. Todas as análises foram feitas no software estatístico IBM SPSS versão 20.0 (IBM Corp., Armonk, NY, EUA). O valor de $p<0,05$ foi adotado para significância estatística.

O trabalho foi aprovado pelo CEP do Hospital sob o CAAE $n$ ${ }^{\circ}:$ 83280217.7.0000.5126.

\section{Resultados}

As - Tabelas 1 e $\mathbf{2}$ apresentam as características gerais da amostra e das lesões. 0 tendão mais frequentemente lesionado foi o supraespinal (SE) (92\%). A maioria dos pacientes apresentou lesão com tamanho até 3 centímetros (60,3\%), Goutallier $<2(77,8 \%)$ e sinal da tangente negativa (90,5\%).

A - Tabela 3 caracteriza o tratamento cirúrgico realizado nos pacientes. Na maioria dos casos $(92,1 \%)$ realizou-se acromioplastia, e em $68,3 \%$ foram usadas duas âncoras na fixação tendão-osso. Aproximadamente metade dos pacientes

Tabela 1 Características dos pacientes

\begin{tabular}{|l|l|l|}
\hline & N & $\%$ \\
\hline Sexo & & \\
\hline Feminino & 36 & 57,1 \\
\hline Masculino & 27 & 42,9 \\
\hline Idade (anos) & $57,2 \pm 9,6$ & \\
\hline Lateralidade & & \\
\hline Direito & 49 & 77,8 \\
\hline Esquerdo & 14 & 22,2 \\
\hline
\end{tabular}

Tabela 2 Características da lesão do manguito rotador

\begin{tabular}{|c|c|c|}
\hline & $\mathrm{N}$ & $\%$ \\
\hline \multicolumn{3}{|c|}{ Tendão acometido } \\
\hline SE & 58 & 92,0 \\
\hline $\mathrm{SE}+\mathrm{IE}$ & 3 & 4,8 \\
\hline $\mathrm{SE}+\mathrm{SC}$ & 2 & 3,2 \\
\hline \multicolumn{3}{|l|}{ Sinal da tangente } \\
\hline Negativo & 57 & 90,5 \\
\hline Positivo & 6 & 9,5 \\
\hline \multicolumn{3}{|l|}{ Goutallier } \\
\hline 0 & 24 & 38,1 \\
\hline 1 & 25 & 39,7 \\
\hline 2 & 14 & 22,2 \\
\hline \multicolumn{3}{|c|}{ Mecanismo da lesão } \\
\hline Degenerativo & 52 & 82,5 \\
\hline Traumático & 11 & 17,5 \\
\hline \multicolumn{3}{|c|}{ Tamanho da lesão } \\
\hline$<1 \mathrm{~cm}$ & 8 & 12,7 \\
\hline $1-3 \mathrm{~cm}$ & 38 & 60,3 \\
\hline $3-5 \mathrm{~cm}$ & 15 & 23,8 \\
\hline$>5 \mathrm{~cm}$ & 2 & 3,2 \\
\hline \multicolumn{3}{|l|}{ Reruptura } \\
\hline Não & 57 & 90,5 \\
\hline Sim & 6 & 9,5 \\
\hline Constant & $92,8 \pm 15,9(62$ a 136$)$ & \\
\hline UCLA & $26,5 \pm 4,3$ (14 a 30$)$ & \\
\hline
\end{tabular}

Abreviaturas: IE, infraEspinal; SC, subescapular; SE, supraespinal. Média \pm desvio-padrão (mínimo - máximo).

Tabela 3 Características do tratamento cirúrgico

\begin{tabular}{|l|l|l|}
\hline & N & $\%$ \\
\hline Âncoras & & \\
\hline 1 & 13 & 20,6 \\
\hline 2 & 43 & 68,3 \\
\hline 3 & 6 & 9,5 \\
\hline 4 & 1 & 1,6 \\
\hline TCLB & & \\
\hline Íntegro & 31 & 49,2 \\
\hline Tenotomia & 30 & 47,6 \\
\hline Tenodese & 2 & 3,2 \\
\hline Acromioplastia & & \\
\hline Não & 5 & 7,9 \\
\hline Sim & 58 & 6,3 \\
\hline Mumford & & \\
\hline Não & 59 & 93,7 \\
\hline Sim & 4 & 6,3 \\
\hline
\end{tabular}

Abreviaturas: TCLB, tendão da cabeça longa do bíceps. 


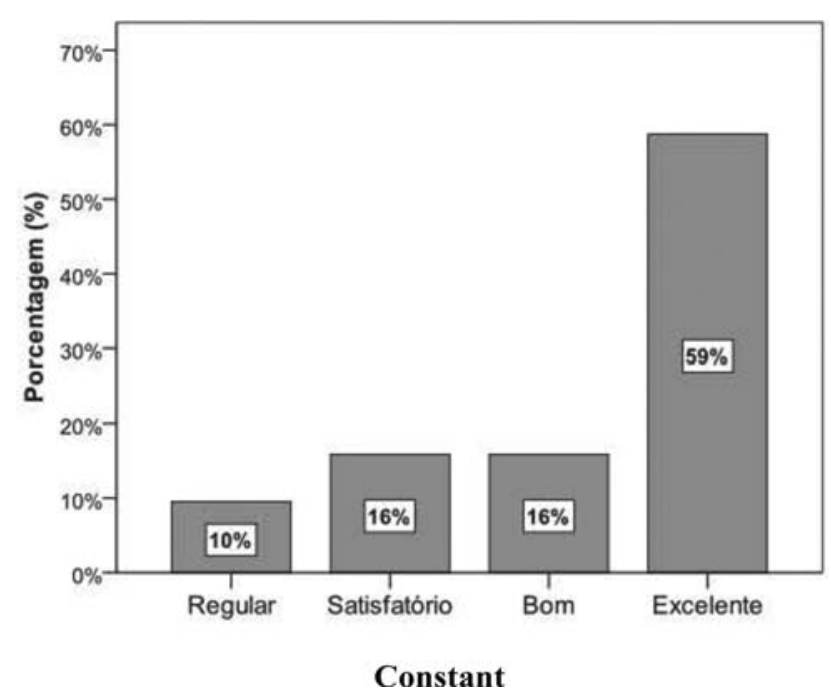

Fig. 1 Classificação funcional dos pacientes segundo escore de Constant.

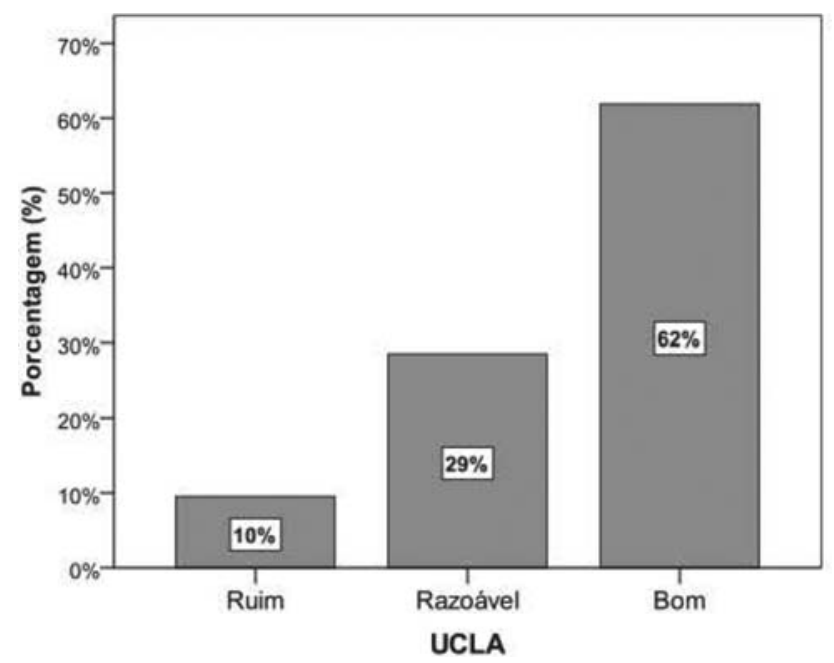

Fig. 2 Classificação funcional dos pacientes segundo escore de UCLA.

apresentaram TCLB íntegro (49,2\%) e apenas 6,3\% realizaram o procedimento de Mumford.

Apenas 9,5\% apresentaram re-ruptura. Na avaliação funcional, foram obtidos valores médios de 26 pontos no escore da UCLA e de 93 pontos no escore de Constant (- Tabela 2). Nas - Figuras 1 e $\mathbf{2}$ pode ser observada a avaliação funcional dos pacientes. Os resultados do Constant foram classificados segundo Boehm ${ }^{18}$ nos subtipos: excelentes (91-100), bons (81-90), satisfatórios (71-80), regulares (61-70) ou maus (< $60)$ de acordo com a pontuação final. Neste trabalho não foram identificados pacientes com mau resultado no escore de Constant $(<60)$. Segundo o escore de Constant, $91 \%$ dos pacientes tiveram seus resultados classificados como satisfatórios, bons ou excelentes, ou seja, apresentaram resultados $>70$ pontos. Os resultados do escore da UCLA foram classificados conforme a metodologia proposta por Ellman et al. ${ }^{19}$ nos subtipos: excelente (34-35), bom (28-33), razoável (21-27) e ruim (00-20) de acordo com a pontuação final. Segundo o escore da UCLA, 62\% dos pacientes tiveram seus resultados classificados como bons, com pontuação $\geq 28$ pontos. Pacientes com resultados classificados como razoáveis totalizaram $29 \%$ e ruins $10 \%$. O presente estudo não identificou pacientes com resultados classificados como excelentes segundo este escore funcional.

As variáveis explicativas associadas à classificação funcional através do UCLA foram: Goutallier, sinal da tangente, tenotomia do TCLB e idade ( - Tabela 4 ). Os pacientes com resultados insatisfatórios no UCLA apresentaram Goutallier $\geq 2$, sinal da tangente positiva, realizaram tenotomia do TCLB sendo que a grande maioria eram mais idosos, com uma média de idade acima de 63 anos. Sob o ponto de vista prático, a associação entre as variáveis explicativas Goutallier, sinal da tangente e tenotomia do TCLB sobre o desfecho funcional no UCLA foi de moderada magnitude, explicando de 9 a 14\% do resultado insatisfatório no escore, de acordo com os valores do V de Cramer. Por outro lado, a variável idade foi de elevada magnitude, sugerindo que ser mais idoso (idade maior que 63 anos) é um forte preditor de resultados insatisfatórios no UCLA.

As variáveis explicativas associadas à classificação funcional através do Constant foram: classificação de Goutallier, sinal da tangente e idade ( - Tabela 4 ). Os pacientes com resultados insatisfatórios no Constant apresentaram Goutallier $\geq 2$, sinal da tangente positiva e eram mais idosos, com uma média de idade acima de 69 anos. Sob o ponto de vista prático, a associação entre as variáveis explicativas Goutallier $\geq 2$ e sinal da tangente positiva sobre o desfecho funcional no Constant foi de moderada magnitude, explicando 7 a $12 \%$ dos resultados insatisfatórios no Constant, de acordo com o V de Cramer. Por outro lado, idade acima de 69 anos foi de elevada magnitude, sugerindo que ser mais idoso é um forte preditor de resultados insatisfatórios no Constant.

\section{Discussão}

Observa-se na literatura poucos estudos que avaliam os escores funcionais de pacientes submetidos ao reparo videoartroscópico do MR com um seguimento pós-operatório mínimo de 10 anos. ${ }^{20-26}$

DeFranco et al. ${ }^{27}$ descreveram que a idade é o principal fator preditivo de integridade estrutural após reparo artroscópico. Em nosso estudo, os pacientes mais jovens obtiveram melhor resultado funcional em comparação com os pacientes mais idosos.

Apesar de não encontrarmos significância estatística, as lesões envolvendo os tendões infraespinal, redondo menor e subescapular, tiveram um grau de insatisfação maior que o grupo envolvendo isoladamente o supraespinal. Esta associação, segundo a literatura ${ }^{2}$, pode ser justificada pelo fato de as lesões póstero-superiores e anterossuperiores estarem associadas a uma lesão de extensão maior $(>3 \mathrm{~cm})$, um grau de infiltração gordurosa mais avançado (Goutallier $>2$ ) e um trofismo muscular de baixa qualidade (sinal da tangente de Zanetti positiva).

Encontramos uma taxa média de 9,5\% de re-ruptura nos exames de RM de controle realizados após 10 anos de reparo do MR, semelhante aos índices encontrados por Sugaya et al. ${ }^{28}$. que evidenciaram $13 \%$ de re-rupturas nas lesões médias e $44 \%$, nas grandes e extensas. 
Tabela 4 Associação entre possíveis fatores de exposição e o desfecho funcional

\begin{tabular}{|c|c|c|c|c|c|c|c|c|c|}
\hline Variável & Categoria & $\begin{array}{l}\text { Constant } \\
\text { satisfatório }\end{array}$ & $\begin{array}{l}\text { Constant } \\
\text { insatisfatório }\end{array}$ & $\begin{array}{l}\text { Valor } \\
\text { de } p\end{array}$ & TE & $\begin{array}{l}\text { UCLA } \\
\text { Satisfatório }\end{array}$ & $\begin{array}{l}\text { UCLA } \\
\text { insatisfatório }\end{array}$ & $\begin{array}{l}\text { Valor } \\
\text { de } p\end{array}$ & $\mathrm{TE}$ \\
\hline & & $(n=57)$ & $(n=6)$ & & & $(n=39)$ & $(n=24)$ & & \\
\hline \multirow[t]{2}{*}{ Sexo } & Feminino $(n=36)$ & $88,9 \%$ & $11,1 \%$ & 0,69 & 0,06 & $58,3 \%$ & $41,7 \%$ & 0,05 & 0,008 \\
\hline & Masculino $(n=27)$ & $92,6 \%$ & $7,4 \%$ & & & $66,7 \%$ & $33,3 \%$ & & \\
\hline \multirow[t]{2}{*}{ Acromioplastia } & Não $(n=5)$ & $80,0 \%$ & $20,0 \%$ & 0,40 & 0,10 & $80,0 \%$ & $20,0 \%$ & 0,64 & 0,11 \\
\hline & $\operatorname{Sim}(n=58)$ & $91,4 \%$ & $8,6 \%$ & & & $60,3 \%$ & $39,7 \%$ & & \\
\hline \multirow[t]{2}{*}{ Mumford } & Não $(n=59)$ & $91,5 \%$ & $8,5 \%$ & 0,34 & 0,14 & $62,7 \%$ & $37,3 \%$ & 0,63 & 0,06 \\
\hline & $\operatorname{Sim}(n=4)$ & $75,0 \%$ & $25,0 \%$ & & & $50,0 \%$ & $50,0 \%$ & & \\
\hline \multirow[t]{2}{*}{$\begin{array}{l}\text { Tamanho } \\
\text { da lesão }\end{array}$} & Até $3 \mathrm{~cm}(\mathrm{n}=46)$ & $88,2 \%$ & $11,8 \%$ & 0,66 & 0,05 & $67,4 \%$ & $32,6 \%$ & 0,14 & 0,19 \\
\hline & $>3 \mathrm{~cm}(\mathrm{n}=17)$ & & & & & $47,1 \%$ & $52,9 \%$ & & \\
\hline \multirow[t]{2}{*}{ Goutallier } & $<2(\mathrm{n}=49)$ & $95,9 \%$ & $4,1 \%$ & $0,02^{*}$ & 0,35 & $71,4 \%$ & $28,6 \%$ & $0,004^{*}$ & 0,37 \\
\hline & $\geq 2(n=14)$ & $71,4 \%$ & $28,6 \%$ & & & $28,6 \%$ & $71,4 \%$ & & \\
\hline Tendões & SE $(n=58)$ & $93,1 \%$ & $6,9 \%$ & 0,07 & 0,30 & $63,8 \%$ & $36,2 \%$ & 0,36 & 0,13 \\
\hline Acometidos & $\mathrm{SE}+\mathrm{IE} / \mathrm{SC}(\mathrm{n}=5)$ & $60,0 \%$ & $40,0 \%$ & & & $40,0 \%$ & $60,0 \%$ & & \\
\hline \multirow[t]{2}{*}{ Tangente } & Negativo $(n=57)$ & $93,0 \%$ & $7,0 \%$ & $0,04^{*}$ & 0,26 & $66,7 \%$ & $33,3 \%$ & $0,03^{*}$ & 0,30 \\
\hline & Positivo $(n=6)$ & $66,7 \%$ & $33,3 \%$ & & & $16,7 \%$ & $83,3 \%$ & & \\
\hline \multirow[t]{2}{*}{ Tenotomia } & Não (n=33) & $97,0 \%$ & $3,0 \%$ & 0,09 & 0,23 & $78,8 \%$ & $21,2 \%$ & $0,004^{*}$ & 0,36 \\
\hline & $\operatorname{Sim}(n=30)$ & $83,3 \%$ & $16,7 \%$ & & & $43,3 \%$ & $56,7 \%$ & & \\
\hline Idade (anos) & & $55,9 \pm 8,9$ & $69,3 \pm 8,8$ & $0,001^{*}$ & 1,50 & $53,7 \pm 8,6$ & $63,0 \pm 8,4$ & $<0,001^{*}$ & 1,09 \\
\hline $\mathrm{N}^{\circ}$ de âncoras & & $1,9 \pm 0,6$ & $2,2 \pm 1,0$ & 0,30 & 0,37 & $1,8 \pm 0,6$ & $2,0 \pm 0,6$ & 0,21 & 0,33 \\
\hline
\end{tabular}

Abreviaturas: SE, supraespinal, IE, infraespinal, SC, subescapular.

(Porcentagens em relação ao total das linhas; ${ }^{*}$ diferença significativa, $p<0,05$; TE: tamanho do efeito. $-\mathrm{d}$ de Cohen [variáveis quantitativas] pequeno: 0,2-0,4; moderado: 0,5-0,7; elevado: $\geq 0,8 ;-V$ de Cramer [variáveis qualitativas] pequeno: 0,1-0,2; moderado: 0,3-0,4; elevado: $\geq 0,5$.

A incidência das lesões foi de etiologia predominantemente degenerativa (82,5\%), de acordo com os achados relatados previamente na literatura. ${ }^{1,2} \mathrm{O}$ número de âncoras utilizadas para o reparo das lesões não influenciou nos resultados dos escores funcionais. Assim como não influenciaram a realização ou não do procedimento de Mumford total e da acromioplastia, dados corroborados por Miyazaki et al. ${ }^{21}$

Os pacientes que não se submeteram à tenotomia do TCLB obtiveram um melhor escore de Constant (satisfatório $97,0 \%$ ), sendo observados os mesmos resultados quanto ao escores UCLA (satisfatório 78,8\%).

O reparo artroscópico nas lesões $<3 \mathrm{~cm}$ obtiveram melhores resultados nas avaliações dos escores de Constant e da UCLA (satisfatórios 91,3\% e 67,4\% respectivamente). Estes índices corroboram com os achados na literatura. ${ }^{26}$ Lesões grandes e extensas, de acordo a Classificação de Cofield, têm associação com piores resultados dos escores funcionais. ${ }^{2}$

No estudo de Collin et al., ${ }^{3}$ foi observado que pacientes com Goutallier $<2$ obtiveram um grau de satisfação na avaliação do escore de Constant em torno de $84,9 \%$. Constatou-se que o grau de infiltração gordurosa pré-operatória é inversamente proporcional ao escore funcional. Em nosso estudo, lesões do manguito com Goutallier $<2$ obtiveram excelentes resultados na avaliação dos escores de Constant e da UCLA (satisfação 95,9\% e 71,4\% respectivamente). $O$ índice de Goutallier $\geq 2$ não contraindica o reparo artroscópico do MR, porém, torna-se uma variável que sugere pior prognóstico, confirmado pelos resultados insatisfatórios encontrados na avaliação dos escores de Constant e UCLA (28,6\% e 71,4\% respectivamente). ${ }^{3}$

O sinal da tangente, quando é negativo, mostrou-se associado a melhores resultados funcionais, com um índice de satisfação no escore de Constant de $93,0 \%$ e de $66,7 \%$ no escore da UCLA, assim como no estudo de Collin et al., ${ }^{3}$ que obteve resultados semelhantes em relação ao escore de Constant, ratificando o melhor prognóstico das lesões quando se há um bom trofismo muscular.

O reparo artroscópico do MR promoveu resultados duráveis e satisfatórios de acordo com o escore de Constant, alcançando excelentes e bons resultados em 75\% da amostra, assim como demonstrado por Galatz et al., ${ }^{20}$ que encontraram $91 \%$ de satisfaçãoem pacientes com seguimento mínimo de 10 anos.

A ausência da avaliação funcional pré-operatória através dos escores da UCLA e de Constant pode ser apontada como uma limitação do presente estudo. O seguimento dos pacientes avaliados no presente estudo permitirá definir se houve variação funcional ao longo do tempo. Outro dado importante a ser considerado é que a maior parte dos pacientes em questão apresentavam lesão isolada do tendão do supraespinal (92\%), o que pode influenciar na avaliação dos resultados.

\section{Conclusão}

O reparo artroscópico do MR mostrou-se efetivo mesmo a longo prazo (seguimento mínimo de 10 anos). 
A idade do paciente no momento da cirurgia, a extensão da lesão, o grau de infiltração gordurosa (Goutallier), e o trofismo muscular (sinal da tangente) foram os preditores de sucesso e/ou insucesso mais fidedignos.

\section{Conflitos de Interesses}

Os autores declaram não haver conflitos de interesses.

\section{Referências}

1 White JJ, Titchener AG, Fakis A, Tambe AA, Hubbard RB, Clark DI. An epidemiological study of rotator cuff pathology using The Health Improvement Network database. Bone Joint J 2014;96-B (03):350-353

2 Veado MAC, Gomes TPO, Pinto RZA. Análise funcional e estrutural do reparo das lesões extensas do manguito rotador. Rev Bras Ortop 2006;41(08):294-301

3 Collin P, Kempf JF, Molé D, et al. Société Française de Chirurgie Orthopédique et Traumatologique (SoFCOT). Ten-Year Multicenter Clinical and MRI Evaluation of Isolated Supraspinatus Repairs. J Bone Joint Surg Am 2017;99(16):1355-1364

4 Godinho GG, França FO, Freitas JM, et al. Avaliação da integridade anatômica por exame de ultrassom e funcional pelo índice de Constant \& Murley do manguito rotador após reparo artroscópico. Rev Bras Ortop 2010;45(02):174-180

5 Rutten MJ, Spaargaren GJ, van Loon T, de Waal Malefijt MC, Kiemeney LA, Jager GJ. Detection of rotator cuff tears: the value of MRI following ultrasound. Eur Radiol 2010;20(02):450-457

6 Ghodadra NS, Provencher MT, Verma NN, Wilk KEW, Romeo AA. Open, mini-open, and all-arthroscopic rotator cuff repair surgery: indications and implications for rehabilitation. J Orthop Sports Phys Ther 2009;39(02):81-89

7 Gartsman GM, Khan M, Hammerman SM. Arthroscopic repair of full-thickness tears of the rotator cuff. J Bone Joint Surg Am 1998; 80(06):832-840

8 Checchia SL, Doneux PS, Miyazaki AN, et al. Avaliação dos resultados obtidos na reparação artroscópica das lesões do manguito rotador. Rev Bras Ortop 2005;40(05):229-238

9 Godinho GG, Souza JM, Bicalho LA. Reparo das rupturas do manguito rotator do ombro pela videoartroscopia cirúrgica: técnica. Rev Bras Ortop 1996;31(04):284-288

10 Levine WN, Barron OA, Yamaguchi K, Pollock RG, Flatow EL, Bigliani LU. Arthroscopic distal clavicle resection from a bursal approach. Arthroscopy 1998;14(01):52-56

11 McLAUGHLIN HL. On the frozen shoulder. Bull Hosp Jt Dis 1951;12 (02):383-393

12 O'Brien SJ, Pagnani MJ, Fealy S, McGlynn SR, Wilson JB. The active compression test: a new and effective test for diagnosing labral tears and acromioclavicular joint abnormality. Am J Sports Med 1998;26(05):610-613

13 Goutallier D, Postel JM, Radier C, Bernageau J, Zilber S. Long-term functional and structural outcome in patients with intact repairs
1 year after open transosseous rotator cuff repair. J Shoulder Elbow Surg 2009;18(04):521-528

14 Zanetti M, Gerber C, Hodler J. Quantitative assessment of the muscles of the rotator cuff with magnetic resonance imaging. Invest Radiol 1998;33(03):163-170

15 Cofield RH. Subscapular muscle transposition for repair of chronic rotator cuff tears. Surg Gynecol Obstet 1982;154(05):667-672

16 Constant CR, Murley AH. A clinical method of functional assessment of the shoulder. Clin Orthop Relat Res 1987;(214): 160-164

17 Amstutz HC, Sew Hoy AL, Clarke IC. UCLA anatomic total shoulder arthroplasty. Clin Orthop Relat Res 1981;(155):7-20

18 Boehm D. Valuation of the Constant score. In: Habermeyer P, Magosch P, Lichtenberg S, eds. Classifications and scores of the shoulder. Heidelberg: Springer; 2006:204

19 Ellman H, Hanker G, Bayer M. Repair of the rotator cuff. End-result study of factors influencing reconstruction. J Bone Joint Surg Am 1986;68(08):1136-1144

20 Galatz LM, Griggs S, Cameron BD, Iannotti JP. Prospective longitudinal analysis of postoperative shoulder function : a ten-year follow-up study of full-thickness rotator cuff tears. J Bone Joint Surg Am 2001;83(07):1052-1056

21 Miyazaki AN, Santos PD, da Silva LA, do Val Sella G, Checchia SL, Yonamine AM. Os bons resultados funcionais do reparo artroscópico das lesões extensas do manguito rotador mantêm-se em longo prazo? Rev Bras Ortop 2015;51(01):40-44

22 Paxton ES, Teefey SA, Dahiya N, Keener JD, Yamaguchi K, Galatz LM. Clinical and radiographic outcomes of failed repairs of large or massive rotator cuff tears: minimum ten-year follow-up. J Bone Joint Surg Am 2013;95(07):627-632

23 Miškulin M, Vrgoč G, Sporiš G, Dulic O, Gavrilovic G, Milanović Z. Single-row arthroscopic cuff repair with double-loaded anchors provides good shoulder function in long-term follow-up. Int Orthop 2015;39(02):233-240

24 Wolf EM, Pennington WT, Agrawal V. Arthroscopic rotator cuff repair: 4- to 10-year results. Arthroscopy 2004;20(01):5-12

25 Nové-Josserand L, Collin P, Godenèche A, Walch G, Meyer N, Kempf JF; SOFCOT. Ten-year clinical and anatomic follow-up after repair of anterosuperior rotator cuff tears: influence of the subscapularis. J Shoulder Elbow Surg 2017;26(10): $1826-1833$

26 Kluger R, Bock P, Mittlböck M, Krampla W, Engel A. Long-term survivorship of rotator cuff repairs using ultrasound and magnetic resonance imaging analysis. Am J Sports Med 2011;39(10): 2071-2081

27 DeFranco MJ, Bershadsky B, Ciccone J, Yum JK, Iannotti JP. Functional outcome of arthroscopic rotator cuff repairs: a correlation of anatomic and clinical results. J Shoulder Elbow Surg 2007;16(06): 759-765

28 Sugaya H, Maeda K, Matsuki K, Moriishi J. Functional and structural outcome after arthroscopic full-thickness rotator cuff repair: single-row versus dual-row fixation. Arthroscopy 2005; 21(11):1307-1316 